\title{
Label-Free Analysis of Protein Aggregation and Phase Behaviour
}

Zenon Toprakcioglu,${ }^{\dagger}$ Pavankumar Challa, ${ }^{\dagger}$ Catherine $\mathrm{Xu}^{\dagger}{ }^{\dagger}$ and Tuomas P. J.

$$
\text { Knowles }{ }^{*, \dagger, t}
$$

$\dagger$ Department of Chemistry, University of Cambridge, Lensfield Road, CB2 1EW, Cambridge UK

$\ddagger$ Cavendish Laboratory, J J Thomson Avenue, CB3 OHE, Cambridge UK

\section{E-mail: tpjk2@cam.ac.uk}

\section{Supplementary Information}

\section{Analysis of kinetic data}

The kinetic model containing the microscopic steps of primary nucleation, fibril elongation, and a multi-step secondary nucleation, have been previously described and are follows:

$$
\begin{gathered}
\frac{d[P]}{d t}=k_{n}\left[m^{n_{c}}\right]+k_{2} \frac{[m]^{n_{2}}}{1+[m]^{n_{2}} / K_{m}}[M] \\
\frac{d[M]}{d t}=2 k_{+}[m][P]
\end{gathered}
$$


where $[\mathrm{P}]$ and $[\mathrm{M}]$ are the number and mass concentrations of aggregates, respectively. The rate constant $\mathrm{k}_{n}, \mathrm{k}_{2}$ and $\mathrm{k}_{+}$symbolise primary nucleation, secondary nucleation and fibril elongation, respectively. [m] is the concentration of monomer, $\mathrm{K}_{m}$ is the Michaelis constant and $\mathrm{n}_{c}$ and $\mathrm{n}_{2}$ are the reaction orders relative to primary and secondary nucleation, respectively.

The kinetic data was first normalised, dividing the ThT fluorescence values at time $\mathrm{t}([\mathrm{M}] \mathrm{t})$ by the corresponding values measured at the plateau $([\mathrm{M}])$, taken as 1.0. The resulting traces were analysed simultaneously with a procedure of global best fitting using the fitting platform amylofit. This employs an integrated rate law obtained from the above equations, as previously described:

$$
\frac{[M]}{[M]_{\infty}}=1-\left(1-\frac{[M]_{0}}{[M]_{\infty}}\right) e^{-k_{\infty} t} \cdot\left(\frac{B_{-}+C_{+} e^{\kappa t}}{B_{+}+C_{+} e^{\kappa t}} \cdot \frac{B_{+}+C_{+}}{B_{-}+C_{+}}\right)^{\frac{k \infty}{\kappa k \infty}}
$$

where the definitions of the parameters are as follows:

$$
\begin{gathered}
\kappa=\sqrt{2[m]_{0} k_{+} \frac{[m]_{0}^{n_{2}} k_{2}}{1+[m]_{0}^{n_{2}} / K_{M}}} \\
\lambda=\sqrt{2 k_{+} k_{n}[m]_{0}^{n_{c}}} \\
C_{ \pm}=\frac{k_{+}[P]_{0}}{\kappa} \pm \frac{k_{+}[M]_{0}}{2[m]_{0} k_{+}} \pm \frac{\lambda^{2}}{2 \kappa^{2}} \\
k_{\infty}=2 k_{+}[P]_{\infty} \\
k_{\infty}^{-}=\sqrt{k_{\infty}^{2}-2 C_{+} C_{-} \kappa^{2}}
\end{gathered}
$$




$$
B_{ \pm}=\frac{k_{\infty} \pm k_{\infty}^{-}}{2 \kappa}
$$




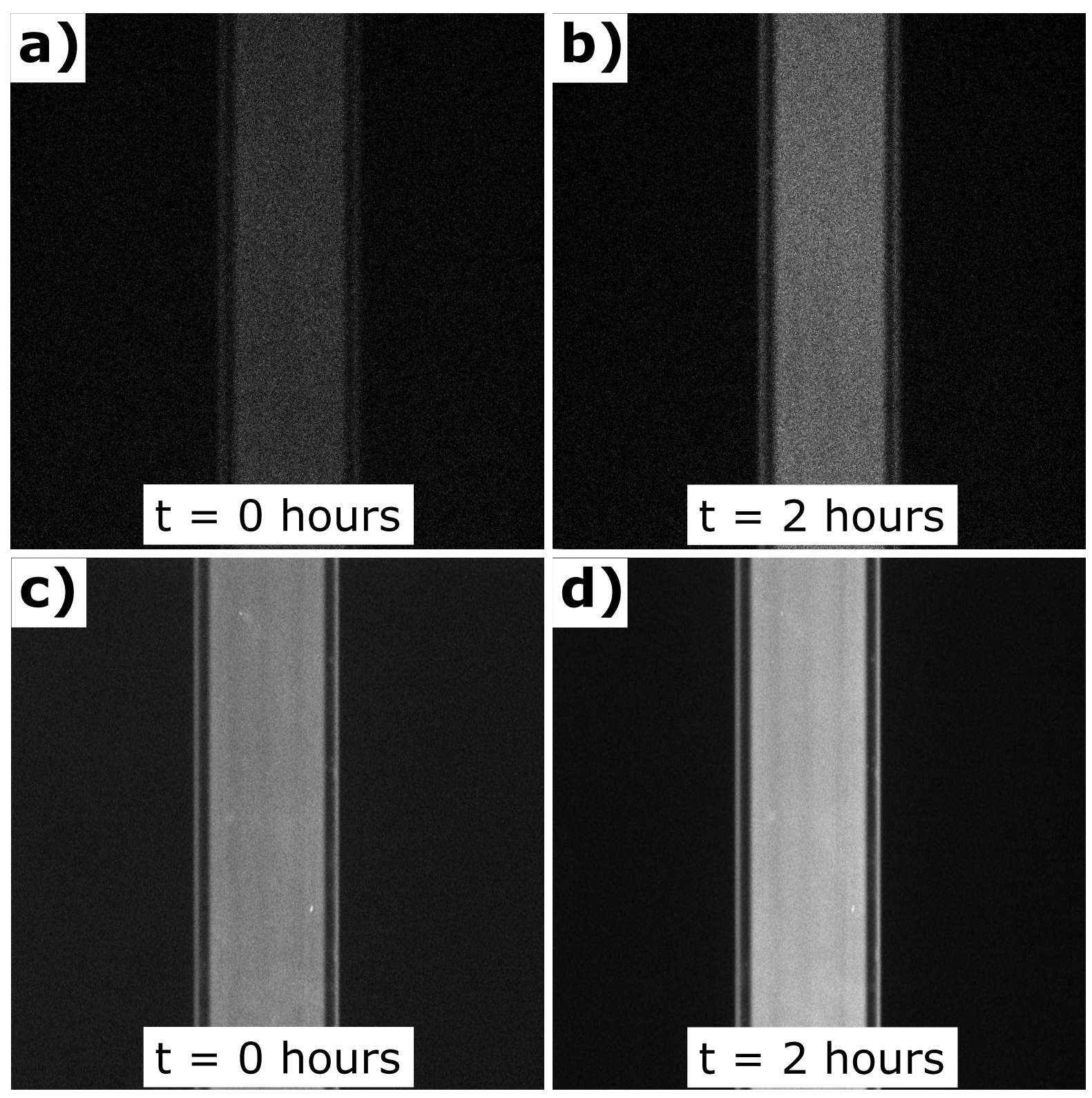

Figure S1: (a-b) Microscopy image sequence of a $30 \mathrm{mg} / \mathrm{mL}$ reconstituted silk fibroin solution monitored using protein intrinsic fluorescence. The monomer containing solution (a) clearly exhibits a lower fluorescent signal than when it has aggregated (b). (c-d) Microscopy image sequence of $30 \mathrm{mg} / \mathrm{mL}$ silk fibroin with ThT solution observed using extrinsic fluorescence. The conversion of monomer (c) to aggregate (d) corresponds with the images taken using intrinsic fluorescence. 


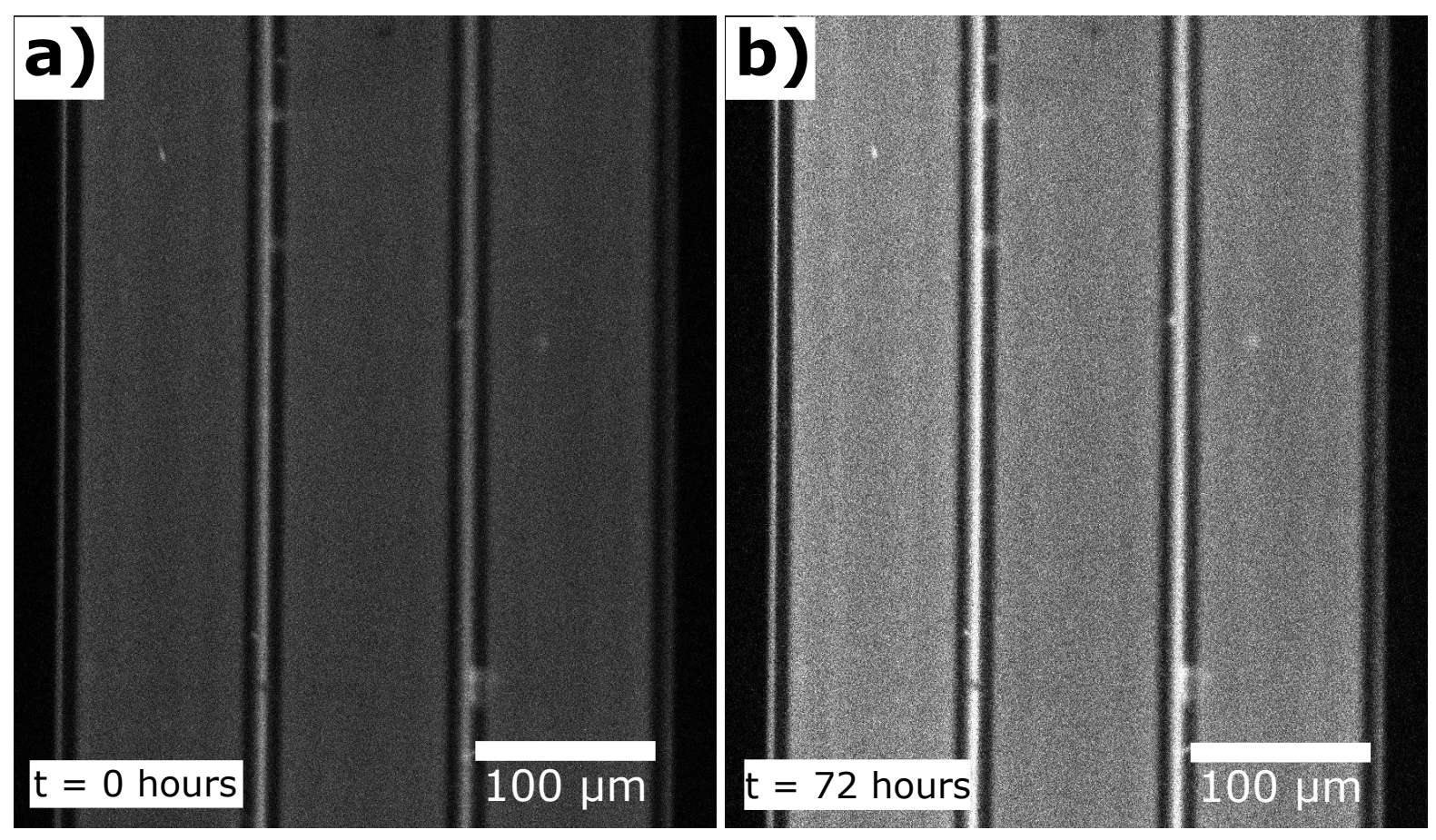

Figure S2: (a-b) Microscopy image sequence of a $80 \mathrm{mg} / \mathrm{mL} \beta$-lactoglobulin solution monitored using protein intrinsic fluorescence. Again it is clear that the monomer containing solution (a) exhibits lower fluorescent signal than in its aggregated form (b). 


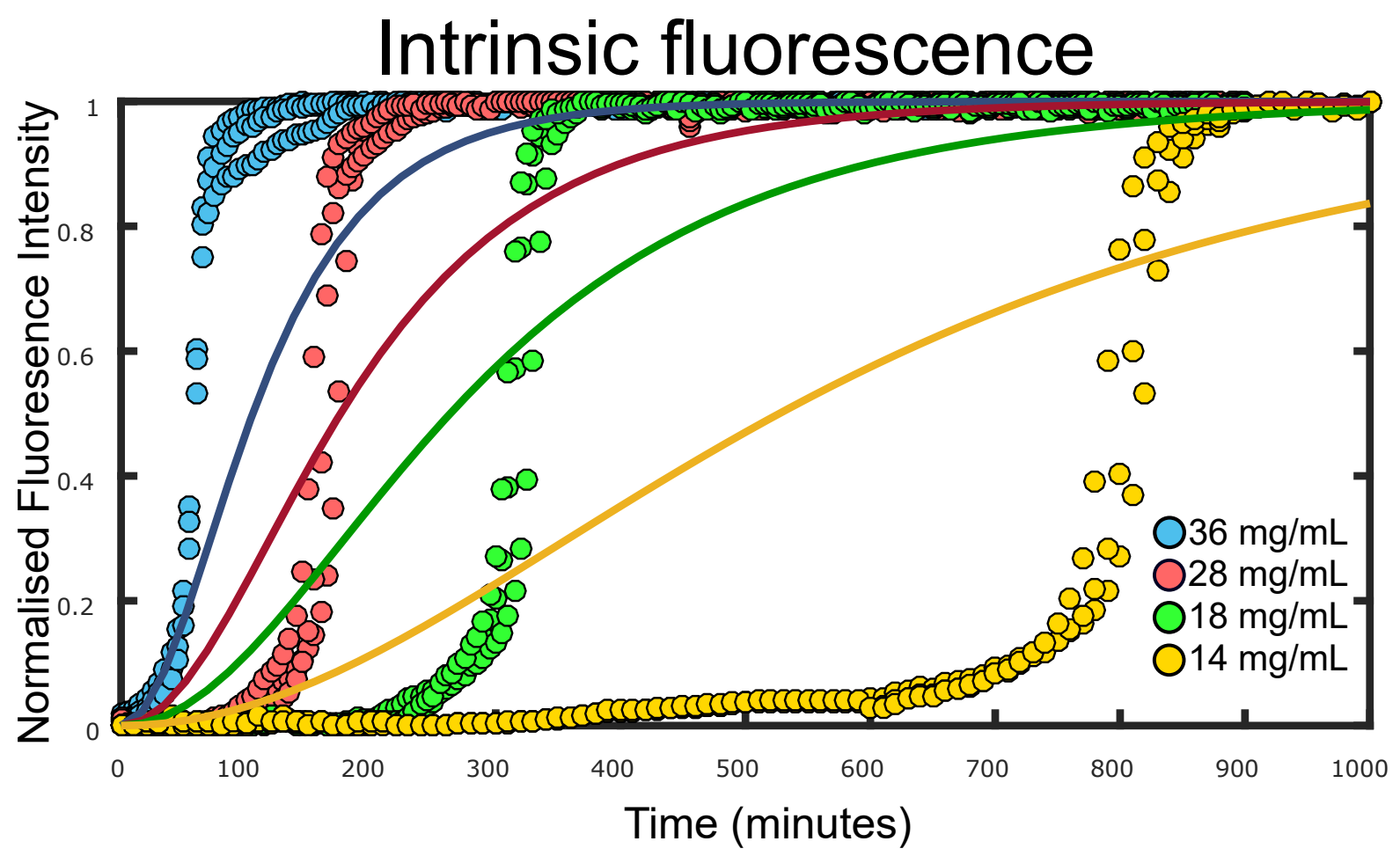

Figure S3: Aggregation kinetics of different silk fibroin concentrations detected by fluorescence emission of tryptophan. For each condition, three replicates are shown and the kinetic data were then globally fitted (solid lines) by assuming primary and elongation nucleation using Amylofit. These misfits clearly indicate that this model is incorrect and a secondary nucleation dominated model is more appropriate. 